\title{
Modeling the acoustic attenuation process of soft tissues
}

\author{
O. ABDEL ALIM, M.M. MOHAMED* and L. SHAAT* \\ Faculty of Engineering, Alexandria University, Alex., Egypt \\ * Medical Research Institute, Alexandria University, Alex., Egypt
}

\begin{abstract}
A mathematical model is developed to simulate attenuation of sofit tissues. A comparison is done between the results of linear model (zero phase and minimum phase) and the noninear model. the nonlinear minimum phase model is verified to be the best model for simulating the attenuation of ultrasound in soft tissues, as compared to the zero phase and the minimum phase linear models.
\end{abstract}

\section{INTRODUCTION}

One approach to ultrasonic characterization of tissue utilizes quantitative measurements of attenuation and backscatter coefficient of normal or diseased tissues. Attenuation measurements have been studied by a number of groups [1-7]. One group begins with an assumption that attenuation $(\alpha)$ increases linearly with frequency (f).

$$
\alpha=\beta \text {. f }
$$

where the single-parameter $\beta$ is used as a measure of attenuation. This model is limited in its ability to represent tissue attenuation $[8,9]$, and therefore leads to systematic, frequency-dependent errors [10]. specifically, the power spectrum of the pulse experiences a downshift in its center frequency which is related to attenuation [11].

In describing an acoustic propagation medium as a linear system, usually only the magnitude of the frequency transfer function is specified. The phase component of the transfer function is often left unspecified because of the difficulty in its measurement, or because direct interpretation of the phase characteristics is not as intuitive as the interpretation of the magnitude function. The phase component has enjoyed increased importance with the recent popularity of time domain signal processing techniques, such as inverse filtering [12], predictive deconvolution [13], or maximum entropy spectral estimation [14], which make some assumptions about the phase properties of a system, usually assumed to be a minimum phase. For minimum-phase systems, the logmagnitude and phase characteristics are related through Hilbert Transform 
[15]. By taking the discrete-time Hilbert Transform of the nonlinear with frequency log magnitude characteristic exhibited by some tissues, we can determine the phase spectrum. The attenuation model presented here is an extension to the work performed by Kuc [16]. The minimum-phase model having a nonlinear characteristic was determined by applying the same procedures described by Kuc [16].

\section{THEORETICAL BASIS}

\section{A. Magnitude transfer function}

Let us consider a section through which the pulse propagates. Let the attenuation coefficient of the tissue, denoted by $\alpha(T, f)$, be a nonlinear function of frequency of the form :

$$
\alpha(T, f)=\alpha_{0}(T) f^{n} \quad \text { for } f>0
$$

where $\mathrm{T}$ is the temperature in ${ }^{\circ} \mathrm{C}, \mathrm{f}$ is the frequency in $\mathrm{MHz}, \alpha$ is the attenuation in $\mathrm{dB} / \mathrm{cm}$, and $\alpha_{0}$ and $\mathrm{n}$ are the magnitude and frequency parameters of the tissue.

The log-magnitude transfer function of a $\mathrm{d} \mathrm{cm}$ thick piece of tissue, denoted by $L(f)$, is then equal to :

$$
L(f)=-\alpha(f, T) d=-\alpha_{0}(T) f^{n} d
$$

The resulting magnitude function, denoted by $|H(f)|$, is then equal to:

$$
|H(f)|=10^{L(f) / 20}=10^{\alpha_{o}(T) f^{n} d / 20}
$$

For the discrete-time (sampled-data) domain, the magnitude characteristic has the above form for $0<f<1 / 2 T_{s}$, where $T_{s}$ is the sampling period. The magnitude characteristic is also an even function of $f$ and is periodic in $f$, with a period $1 / T_{s}$.

B. Discrete-Time Hilbert Transform

For a minimum-phase filter, it is well known that the log magnitude and phase functions form a Hilbert Transform [15]. For the discrete-time domain, the Hilbert Transform of the log-magnitude has the form :

$$
\text { phase }|H(\omega)|=(1 / 2 \pi) P_{-\pi}^{\prime} \int^{\pi} \log |H(\theta)| \cot ((\theta-\omega) / 2) d \theta
$$

where $\omega=2 \pi f T_{s}$ and the symbol $P^{\prime}$ denotes the Cauchy principal value of the integral [15]. If the Cauchy function is zero, the value of the function becomes infinite and the integral must be evaluated through a limiting procedure.

\section{EXPERIMENTAL. VERIFICATION AND RESULTS}

To verify the minimum-phase model, a pulse-reflection experiment was performed on bovine liver, human breast, and cancer breast tissues submerged in water. The transducer is immersed in a rectangular temperature controlled water tank. The transducer is utilized as both a pulse transmitter and receiver. Stainless steel reflector is located in the focal region of the transducer and aligned for normal wave incidence. The sample is interposed between the transducer and the reflector. The pulses reflected from the stainless steel reflector without sample, $p_{r}$, and those reflected with the tissue sample interposed between the transducer and reflector, $p_{s}$, are measured.

To determine the $\log$ magnitude transfer function of the soft tissue sample, the stainless steel reflection power spectrum (without sample), 
$S_{r}(f)$, and that with the sample interposed between the transducer and the stainless steel reflector. $S_{s}(f)$, are calculated as the squared magnitude of the Fourier Transform of the respective pulse sequence. The frequency dependence of the attenuated signal can then determined by subtracting the soft tissue sample log spectrum from that of the stainless steel.

Table 1 shows the values of nonlinear attenuation coefficient $n$ and $\alpha_{0}$ and the value of linear attenuation coefficient $B$ for bovine liver, normal breast and cancer breast. Using these parameters, the log magnitude transfer function $L(f)$ of a $d \mathrm{~cm}$ thick piece of tissue is then calculated from equation (3), and the resulting magnitude function $|H(f)|$ is obtained from equation (4). The minimum-phase unit-sample response of the digital filter models for the bovine liver, normal female breast, and cancer breast tissues are calculated from the log-magnitude transfer function having the same attenuation magnitude parameter $\alpha$, and frequency parameter $n$ of the respective tissue, and from the phase determined by Discrete Hilbert Transform (DHT).

Table I : Attenuation coefficients $\left(n, \alpha_{0}\right.$ and $\beta$ ) and normalized RMS. error for minimum phase predictions.

\begin{tabular}{|c|c|c|c|c|c||}
\hline SAMLE & $\begin{array}{c}\alpha o \\
\left(d b / \mathrm{cm}^{\prime} M z^{n}\right)\end{array}$ & $n$ & $\left.\begin{array}{c}\beta \\
(d B / c m . M H z\end{array}\right)$ & $\begin{array}{c}\text { ms. error } \\
\text { nonlinear } \\
\text { min. phase }\end{array}$ & $\begin{array}{c}\text { ms. error } \\
\text { linear min. } \\
\text { phase }\end{array}$ \\
\hline \hline Bovine liver & 0.6760 & 1.3206 & 0.8440 & $16.3 \%$ & $54.9 \%$ \\
\hline Normal breast & 1.3740 & 1.0486 & 1.4211 & $17.3 \%$ & $19.1 \%$ \\
\hline Cancer breast & 3.2187 & 0.7786 & 2.7608 & $17.3 \%$ & $63.4 \%$ \\
\hline
\end{tabular}
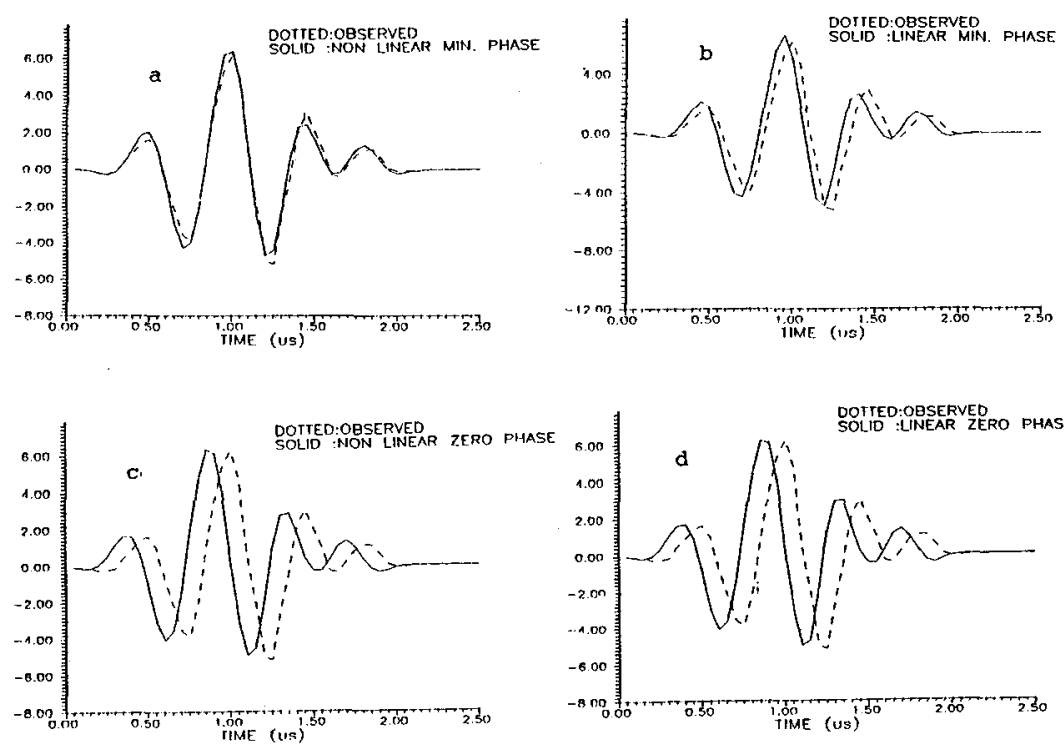

Figure (1) : Comparison of predicted \& reflections

a) Bovine liver-non linear min. phase.

b) Bovine liver-linear min. phase.

c) Bovine liver-linear zero phase.

d) Bovine liver-non linear zero phase. 


\section{COMPARISON BETWEEN DIFFERENT MODELS}

To verify the accuracy of the model, the stainless steel surface reflection $p_{r}$ is convoluted with the unit sample response to predict the form of the stainless steel reflection with sample, $p_{s}$, for linear and non linear minimum phase models. The model is accurate when the predicted and observed wave forms are identical.

The results shown in Figure 1a, indicate that the nonlinear minimum phase is almost exact, and presents a better model than the linear minimum phase, Figure $1 \mathrm{~b}$.

Table 1 shows the normalized rms. error for the nonlinear and linear minimum phase predictions. It is noted that the rms. error for normal breast is almost the same for linear and nonlinear models, since the non linearity presented by $\mathrm{n}$ is close to 1 .

For comparison, a digital filter model having a zero phase characteristic is implemented. Assuming a zero phase characteristic is equivalent to assuming that the real value magnitude function, $|H(f)|$, is the entire complex value function $H(f)$, i.e. $H(f)$ is taken as the real part, and the imaginary part is taken as zero. In this case, the unitsample response of the filter with zero phase is simply the Inverse Fourier Transform of equation (4). The resulting zero-phase unit-sample response is non causal, having non-zero values for negative time. But, by adding a sufficient amount of negative linear phase, the finite-duration unit-sample response can be delayed and made causal [16].

Applying the same verification procedures as above to the linear and non linear zero-phase models produces the prediction of the reflected pulses for the liver as shown in Figures 1 , (c \& d). The mismatch in the assumed phase characteristic of the zero phase filter model results in a poor prediction.

\section{CONCLUSION}

The above shown results indicate the superiority of the non linear minimum phase digital model over the other models for modeling acoustic attenuation for soft tissues.

\section{REFERENCES}

1. Walach, E., et.al IEEE Trans. Biomed. Eng., 33, 637 (1986).

2. Parker, K.J. Soc. Amer., 80, 727 (1986).

3. Parker, K.J., et.al Ultrason. Med. Biol., 14, 127 (1988).

4. Leeman, S., et.al IEEE Trans. Sonics Ultrason 31, 352 (1984).

5. Kuc, R. and Schwartz, M. IEEE Trans. sonics Ultrason. 26, 353 (1979).

6. Narayana, P.A., et.al J.Acoust. Soc. Amer. 76, 1 (1984).

7. Garra, B.S., et.al Radiology, 162, 61 (1987).

8. Parker, K.J. and Tuthill, T.A. IEEE Trans. Biomed. Eng. 33, 453 (1986).

9. Parker, K.J. Ultrasound Med. Biol., 9, 363 (1983).

10. Narayana, P.A. and Ophir, J., Ultrason. Med. Biol. 9, 357 (1983). 11. Narayana, P.A. and Ophir, J. Ultrason. Med. Biol. 10, 715 (1984).

12. Treitel, S. and Robinson, E.A. IEEE Trans. Geosci. Electron, 4, 25 $(1966)$.

13. Makhaul, J. Proc. IEEE, 63, 561 (1975) (Correction in 1976).

14. Gotowaski, P.R., et.al IEEE Trans. Geosci. Electron, 16, 80, (1978).

15. Oppenheim, A. and Schafer, R. Prentica-Hall, Englewood Cliffs (1975).

16. Kuc, R. Ultrasonic Imaging, 6, 24 (1984). 